\title{
Hausdorff dimension of sums of sets with themselves
}

\author{
by \\ T. W. KÖRNER (Cambridge)
}

\begin{abstract}
There is no non-trivial constraint on the Hausdorff dimension of sums of a set with itself.
\end{abstract}

1. Introduction. Throughout this paper we work on the circle $\mathbb{T}=\mathbb{R} / \mathbb{Z}$ but similar results hold on $\mathbb{T}^{n}$ and $\mathbb{R}^{n}$. All measures will be Borel measures and $|I|$ denotes the length of an interval $I$. If $E \subseteq \mathbb{T}$ we write

$$
E_{[n]}=\underbrace{E+\cdots+E}_{n}=\left\{\sum_{j=1}^{n} e_{j}: e_{j} \in E\right\} .
$$

Readers unused to the convention should also note that, if $E \subseteq \mathbb{T}$ and $\delta>0$, we write

$$
E+[-\delta, \delta]=\{e+t: e \in E,|t| \leq \delta\} .
$$

Definition 1.1. The Hausdorff dimension of an $E \subseteq \mathbb{T}$ is the infimum of the set consisting of 0 and all those $\alpha \geq 0$ having the following property: Given any $\varepsilon>0$, we can find a collection $\mathcal{I}$ of closed intervals such that

$$
\bigcup_{I \in \mathcal{I}} I \supseteq E \text { and } \quad \sum_{I \in \mathcal{I}}|I|^{\alpha} \leq \varepsilon .
$$

Hausdorff dimension is translation invariant and so the Hausdorff dimension of $E_{[n+1]}$ must be at least as large as the Hausdorff dimension of $E_{[n]}$.

The object of this paper is to prove the following theorem.

THEOREM 1.2. Given a sequence $\alpha_{j}$ with $0 \leq \alpha_{j} \leq \alpha_{j+1}<1$, we can find a closed set $E$ such that $E_{[j]}$ has Hausdorff dimension $\alpha_{j}$ for each $j \geq 1$.

Our argument can be easily adapted to prove the following variation.

TheOrem 1.3. Given a sequence $\alpha_{j}$ with $0 \leq \alpha_{j} \leq \alpha_{j+1} \leq 1$, we can find a closed set $E$ such that $E_{[j]}$ has Hausdorff dimension $\alpha_{j}$ for each $j \geq 1$. If $\alpha_{k+1}=1$ we can choose $E$ so that, in addition, $E_{[k+1]}=\mathbb{T}$ but $E_{[k]}$

2000 Mathematics Subject Classification: Primary 28A78.

Key words and phrases: Hausdorff dimension. 
has Lebesgue measure zero. Alternatively, we can choose $E$ so that $E_{[j]}$ has Lebesgue measure zero for all $j$.

2. Preliminaries. It is often relatively easy to prove upper bounds for the Hausdorff dimension of a set by providing a suitable cover of intervals, but relatively hard to prove lower bounds. We obtain lower bounds by using the following well known result (see Section 6.2 of Falconer's elegant text [1]).

TheOREM 2.1. Let $E$ be a bounded closed set and $1>\alpha \geq 0$. If we can find a probability measure $\mu$ with support contained in $E$ such that

$$
\iint_{\mathbb{T}^{2}} \frac{d \mu(x) d \mu(y)}{|x-y|^{\alpha}}<\infty
$$

then the Hausdorff dimension of $E$ is at least $\alpha$.

We shall use an elementary observation.

Lemma 2.2. Let $1>\beta>\alpha \geq 0$. Let $g$ be a piecewise continuous positive function. If we define $g_{n}$, for $n^{1-1 / \beta} \geq 2$, by the conditions

$$
g_{n}(x)= \begin{cases}a_{r, n} & \text { if }\left|x-r n^{-1}\right| \leq n^{-1 / \beta}, r \in \mathbb{Z} \\ 0 & \text { otherwise }\end{cases}
$$

where

$$
a_{r, n}=\int_{(r-1 / 2) / n}^{(r+1 / 2) / n} g(x) d x
$$

then

$$
\iint_{\mathbb{T}^{2}} \frac{g_{n}(x) g_{n}(y)}{|x-y|^{\alpha}} d x d y \rightarrow \iint_{\mathbb{T}^{2}} \frac{g(x) g(y)}{|x-y|^{\alpha}} d x d y
$$

as $n \rightarrow \infty$.

Proof. We show that, in fact,

$$
\int_{\mathbb{T}} \frac{g_{n}(x)}{|x-y|^{\alpha}} d x \rightarrow \int_{\mathbb{T}} \frac{g(x)}{|x-y|^{\alpha}} d x
$$

uniformly in $y$. To this end, observe that, if $10^{-1}>\delta>0$, then

$$
\int_{|x-y| \geq \delta} \frac{g_{n}(x)}{|x-y|^{\alpha}} d x \rightarrow \int_{|x-y| \geq \delta} \frac{g(x)}{|x-y|^{\alpha}} d x
$$

uniformly as $n \rightarrow \infty$. Next note that

$$
\int_{|x-y| \leq \delta} \frac{g(x)}{|x-y|^{\alpha}} d x \leq\|g\|_{\infty} \int_{|x| \leq \delta}|x|^{\alpha} d x=\frac{2\|g\|_{\infty}}{1-\alpha} \delta^{1-\alpha} \rightarrow 0
$$


as $\delta \rightarrow 0$. Finally observe that simple estimates give $\left|a_{r, n}\right| \leq 2 n^{1 / \beta-1}\|g\|_{\infty}$ and

$$
\begin{aligned}
\int_{|x-y| \geq \delta} \frac{g_{n}(x)}{|x-y|^{\alpha}} d x & \leq 2\|g\|_{\infty} n^{1 / \beta-1} \int_{|x| \leq 8 n^{-1 / \beta}} \frac{1}{|x|^{\alpha}} d x+2\|g\|_{\infty} \sum_{1 \leq r \leq n \delta} \frac{n^{\alpha}}{|r|^{\alpha}} \\
& \leq 2\|g\|_{\infty} n^{1 / \beta-1} \frac{8^{1-\alpha}}{1-\alpha} n^{-(1-\alpha) / \beta}+\frac{4\|g\|_{\infty}}{1-\alpha} \delta^{1-\alpha} \\
& \leq \frac{16\|g\|_{\infty}}{1-\alpha} n^{\alpha / \beta-1}+\frac{4\|g\|_{\infty}}{1-\alpha} \delta^{1-\alpha} \rightarrow 0
\end{aligned}
$$

as $\delta \rightarrow 0$ and $n \rightarrow \infty$.

We shall prove Theorem 1.2 by a Baire category argument. We use the Hausdorff metric $d_{\mathcal{F}}$ defined in the next lemma.

Definition 2.3. Consider the space $\mathcal{F}$ of non-empty closed subsets of $\mathbb{T}$. We set

$$
d_{\mathcal{F}}(E, F)=\sup _{e \in E} \inf _{f \in F}|e-f|+\sup _{f \in F} \inf _{e \in E}|e-f| .
$$

It is well known that $\left(\mathcal{F}, d_{\mathcal{F}}\right)$ is a complete metric space. (See, for example, [2, Chapter II, $\S 21$, VII and Chapter III, §33, IV].) We need the following observation.

LEMMA 2.4. Let $j$ be a strictly positive integer and let $K>0$. Suppose that $E(n)$ is a closed subset of $\mathbb{T}$ such that there exists a probability measure $\mu_{n}$ with

$$
\operatorname{supp} \mu_{n} \subseteq E(n)_{[j]} \quad \text { and } \quad \iint_{\mathbb{T}^{2}} \frac{d \mu_{n}(x) d \mu_{n}(y)}{|x-y|^{\alpha}} \leq K .
$$

Then, if $E \in \mathcal{F}$ and $d_{\mathcal{F}}(E(n), E) \rightarrow 0$ as $n \rightarrow \infty$, there exists a probability measure $\mu$ with

$$
\operatorname{supp} \mu \subseteq E_{[j]} \quad \text { and } \quad \iint_{\mathbb{T}^{2}} \frac{d \mu(x) d \mu(y)}{|x-y|^{\alpha}} \leq K .
$$

Proof. Since the set of probability measures is weak-star compact, we may suppose, by extracting a subsequence, that $\mu_{n} \rightarrow \mu$ weak-star. Since $d_{\mathcal{F}}(E(n), E) \rightarrow 0$ we have $d_{\mathcal{F}}\left(E(n)_{[j]}, E_{[j]}\right) \rightarrow 0$ and $\operatorname{supp} \mu \subseteq E_{[j]}$. Since

$$
\iint_{\mathbb{T}^{2}} \frac{d \mu(x) d \mu(y)}{|x-y|^{\alpha}} \leq \liminf _{n \rightarrow \infty} \iint_{\mathbb{T}^{2}} \frac{d \mu_{n}(x) d \mu_{n}(y)}{|x-y|^{\alpha}} \leq K
$$

we are done.

LEMmA 2.5. Let $0 \leq \alpha_{j} \leq \alpha_{j+1}<1$ and $K_{j}>0$. Let $\mathcal{G}$ be the collection of closed sets $E$ such that, for each $j \geq 1$, there exists a probability 
measure $\mu_{j}$ with

$$
\operatorname{supp} \mu_{j} \subseteq E_{[j]} \quad \text { and } \quad \iint_{\mathbb{T}^{2}} \frac{d \mu_{j}(x) d \mu_{j}(y)}{|x-y|^{\alpha_{j}}} \leq K_{j} .
$$

Then $\mathcal{G}$ is a closed subset of $\left(\mathcal{F}, d_{\mathcal{F}}\right)$.

Proof. Immediate from Lemma 2.4.

As matters stand, $\mathcal{G}$ could be empty. However, if $E$ is the union of a finite collection of closed intervals (for example if $E=\mathbb{T}$ ), then, if we take $\tau$ to be the uniform probability measure on $E$ and set

$$
K_{j}=1+\iint_{\mathbb{T}^{2}} \frac{d \tau(x) d \tau(y)}{|x-y|^{\alpha_{j}}},
$$

we will have $E \in \mathcal{G}$.

From now on, the $\alpha_{j}$ will form a fixed sequence satisfying the conditions of Lemma 2.5 and the $K_{j}$ will be a fixed sequence chosen so that

$$
K_{j}>\iint_{\mathbb{T}^{2}} \frac{1}{|x-y|^{\alpha_{j}}} d x d y .
$$

If $d_{\mathcal{G}}$ is the restriction of the metric $d_{\mathcal{F}}$ to the space $\mathcal{G}$, we now know that $\left(\mathcal{G}, d_{\mathcal{G}}\right)$ is complete and non-empty. Theorem 1.2 thus follows from its Baire category version.

TheOrem 2.6. The set of $E \in \mathcal{G}$ such that $E_{[j]}$ has Hausdorff dimension $\alpha_{j}$ for all $j \geq 1$ is of second category in $\left(\mathcal{G}, d_{\mathcal{G}}\right)$.

We can now reduce the proof of Theorem 2.6 to the following lemma.

Lemma 2.7. Let $\eta>0$ and $n \geq 1$. Then the set $\mathcal{E}$ of $E \in \mathcal{G}$ such that there exist a finite collection $\mathcal{I}$ of closed intervals with

$$
\bigcup_{I \in \mathcal{I}} I \supseteq E_{[n]} \quad \text { and } \quad \sum_{I \in \mathcal{I}}|I|^{\alpha_{n}+\eta}<\eta
$$

is dense in $\left(\mathcal{G}, d_{\mathcal{G}}\right)$.

We supplement Lemma 2.7 with a simple observation.

Lemma 2.8. Let $\eta>0$ and $n \geq 1$. Then the set $\mathcal{E}$ of $E \in \mathcal{G}$ such that there exist a finite collection $\mathcal{I}$ of closed intervals with

$$
\bigcup_{I \in \mathcal{I}} I \supseteq E_{[n]} \quad \text { and } \quad \sum_{I \in \mathcal{I}}|I|^{\alpha_{n}+\eta}<\eta
$$

is open in $\left(\mathcal{G}, d_{\mathcal{G}}\right)$.

Proof. Observe that if

$$
\bigcup_{I \in \mathcal{I}} I \supseteq E_{[n]} \quad \text { and } \quad \sum_{I \in \mathcal{I}}|I|^{\alpha_{n}+\eta}<\eta,
$$


then, if $\theta>0$ is small enough,

$$
\sum_{I \in \mathcal{I}}|(I+[-\theta, \theta])|^{\alpha_{n}+\eta}<\eta \quad \text { and } \bigcup_{I \in \mathcal{I}}(I+[-\theta, \theta]) \supseteq F_{[n]}
$$

whenever $d(F, E)<\theta / n$. Thus $\mathcal{E}$ is open.

Proof of Theorem 2.6 from Lemma 2.7. Let us write $\mathcal{E}(j, m)$ for the set of $E \in \mathcal{G}$ such that there exist a finite collection $\mathcal{I}(j, m)$ of closed intervals with

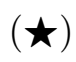

$$
\bigcup_{I \in \mathcal{I}(j, m)} I \supseteq E_{[j]} \quad \text { and } \sum_{I \in \mathcal{I}(j, m)}|I|^{\alpha_{j}+1 / m}<1 / m
$$

By Lemmas 2.7 and $2.8, \mathcal{I}(j, m)$ is open and dense, so

$$
\mathcal{H}=\bigcap_{j=1}^{\infty} \bigcap_{m=1}^{\infty} \mathcal{E}(j, m)
$$

is of second category in $\left(\mathcal{G}, d_{\mathcal{G}}\right)$.

If $E \in \mathcal{H}$ and $j \geq 1$ then the definition of $\mathcal{G}$ together with Theorem 2.1 tells us that $E_{[j]}$ has Hausdorff dimension at least $\alpha_{j}$. However, $E_{[j]}$ also obeys the conditions given in $(\star)$, so $E_{[j]}$ has Hausdorff dimension at most $\alpha_{j}$ and we are done.

3. Proof of the main lemma. Up to now, the use of Baire category has allowed the argument to flow smoothly. We get our hands dirty in the statement and proof of Lemma 2.7, which forms the central step of our argument.

As often happens with complicated constructions, the reader may find it easiest first to get a rough idea of how Lemma 3.2 follows from Lemma 3.1 and Lemma 2.7 from Lemma 3.2. It may also be helpful to run through the proof of Lemma 3.1 with $m=0$ and then look at how much of the proof of Lemma 2.7 goes through with $m=0$.

LEMMA 3.1. Let $\delta, \eta>0$, and $n, m \geq 1$. Write

$$
\Lambda=\{r: n+m \geq r \geq 1\}
$$

Suppose $E_{1}, \ldots, E_{n+m}$ are each the finite union of non-trivial closed intervals such that whenever $L \subseteq \Lambda$, and $L$ contains $j$ elements with $n \geq j \geq 1$, there exists a piecewise continuous positive $g_{L}: \mathbb{T} \rightarrow \mathbb{R}$ with

$$
\operatorname{supp} g_{L} \subseteq\left(\bigcup_{r \in L} E_{r}\right)_{[j]}, \quad \int_{\mathbb{T}} g_{L}(x) d x=1, \quad \iint_{\mathbb{T}^{2}} \frac{g_{L}(x) g_{L}(y)}{|x-y|^{\alpha_{j}}} d x d y<K_{j} .
$$

Then, given any subset $P$ of $\Lambda$ containing exactly $n$ members, we can find $\widetilde{E}_{1}, \ldots, \widetilde{E}_{n+m}$, each the finite union of non-trivial closed intervals, together with piecewise continuous positive functions $\widetilde{g}_{L}: \mathbb{T} \rightarrow \mathbb{R}$ corresponding to 
every $L \subseteq \Lambda$ containing at least one and at most $n$ elements, having the following properties:

(i) $d_{\mathcal{F}}\left(E_{r}, \widetilde{E}_{r}\right)<\delta$ for all $1 \leq r \leq n+m$. (ii) $\sum_{r \in Q} \widetilde{E}_{r} \supseteq \sum_{r \in Q} E_{r}$ whenever $Q \subseteq \Lambda$ contains at least $n+1$ mem-

(iii) We can find a finite collection $\mathcal{I}(P)$ of intervals such that

$$
\bigcup_{I \in \mathcal{I}(P)} I \supseteq\left(\bigcup_{r \in P} \widetilde{E}_{r}\right)_{[n]} \text { and } \sum_{I \in \mathcal{I}(P)}|I|^{\alpha_{n}+\eta}<\eta\left(\begin{array}{c}
n+m \\
n
\end{array}\right)^{-1} \text {. }
$$

(iv) If $L \subseteq \Lambda$ contains $j$ points with $n \geq j \geq 1$, then

$$
\operatorname{supp} \widetilde{g}_{L} \subseteq\left(\bigcup_{r \in L} \widetilde{E}_{r}\right)_{[j]}, \quad \int_{\mathbb{T}} \widetilde{g}_{L}(x) d x=1, \quad \iint_{\mathbb{T}^{2}} \frac{\widetilde{g}_{L}(x) \widetilde{g}_{L}(y)}{|x-y|^{\alpha_{j}}} d x d y<K_{j} .
$$

Proof. Let $d(x, E)=\inf _{e \in E}|x-e|$. We set $\beta_{n}=\alpha_{n}+\eta / 2$, take

$$
\widetilde{E}_{r}= \begin{cases}E_{r}+[-\delta / 2, \delta / 2] & \text { if } r \notin P, \\ \bigcup_{d\left(q / N, E_{r}\right) \leq \delta / 2}\left[q / N-N^{-1 / \beta_{n}}, q / N+N^{-1 / \beta_{n}}\right] & \text { if } r \in P,\end{cases}
$$

and define $\widetilde{g}_{L}$ by the conditions

$$
\widetilde{g}_{L}(x)= \begin{cases}a_{L, q, N} & \text { if }|x-q / N| \leq N^{-1 / \beta_{n}} \\ 0 & \text { otherwise }\end{cases}
$$

where

$$
a_{L, q, N}=\int_{(q-1 / 2) / N}^{(q+1 / 2) / N} g_{L}(x) d x .
$$

Provided the integer $N$ is large enough, conclusions (i) and (ii) hold automatically whilst (iv) follows from Lemma 2.2. Finally, we observe that

$$
\bigcup_{q=1}^{N}\left[q / N-n N^{-1 / \beta_{n}}, q / N+n N^{-1 / \beta_{n}}\right] \supseteq\left(\bigcup_{r \neq p} \widetilde{E}_{r}\right)_{[n]}
$$

and

$$
\begin{aligned}
\sum_{q=1}^{N} \mid\left[q / N-n N^{-1 / \beta_{n}},\right. & \left.q / N+n N^{-1 / \beta_{n}}\right]\left.\right|^{\alpha_{n}+\eta} \\
& =N \cdot\left(2 n N^{-1 / \beta_{n}}\right)^{\alpha_{n}}=(2 n)^{\alpha_{n}+\eta} N^{-\eta /\left(2 \beta_{n}\right)}<\eta
\end{aligned}
$$

provided that $N$ is large enough.

It is easy to deduce a very slightly stronger result.

Lemma 3.2. Let $\delta, \eta>0$, and $n, m \geq 1$. Write

$$
\Lambda=\{r: n+m \geq r \geq 1\}
$$


Suppose $E_{1}, \ldots, E_{n+m}$ are each the finite union of non-trivial closed intervals such that whenever $L \subseteq \Lambda$, and $L$ contains $j$ elements with $n \geq j \geq 1$, there exists a piecewise continuous positive $g_{L}: \mathbb{T} \rightarrow \mathbb{R}$ with

$$
\operatorname{supp} g_{L} \subseteq\left(\bigcup_{r \in L} E_{r}\right)_{[j]}, \quad \int_{\mathbb{T}} g_{L}(x) d x=1, \quad \iint_{\mathbb{T}^{2}} \frac{g_{L}(x) g_{L}(y)}{|x-y|^{\alpha_{j}}} d x d y<K_{j} .
$$

Then we can find $\widetilde{E}_{1}, \ldots, \widetilde{E}_{n+m}$, each the finite union of non-trivial closed intervals, together with piecewise continuous positive functions $\widetilde{g}_{L}: \mathbb{T} \rightarrow$ $\mathbb{R}$ corresponding to every $L \subseteq \Lambda$ containing at least one and at most $n$ elements, having the following properties:

(i) $d_{\mathcal{F}}\left(E_{r}, \widetilde{E}_{r}\right)<\delta$ for all $1 \leq r \leq n+m$.

(ii) $\sum_{r \in Q} \widetilde{E}_{r} \supseteq \sum_{r \in Q} E_{r}$ whenever $Q \subseteq \Lambda$ contains at least $n+1$ mem-

(iii) Whenever $P \subseteq \Lambda$ contains exactly $n$ members there exists a finite collection $\mathcal{I}(P)$ of intervals such that

$$
\bigcup_{I \in \mathcal{I}(P)} I \supseteq\left(\bigcup_{r \in P} \widetilde{E}_{r}\right)_{[n]} \text { and } \sum_{I \in \mathcal{I}(P)}|I|^{\alpha_{n}+\eta}<\delta\left(\begin{array}{c}
n+m \\
m
\end{array}\right)^{-1} .
$$

(iv) If $L \subseteq \Lambda$ contains $j$ points with $n \geq j \geq 1$, then

$$
\operatorname{supp} \widetilde{g}_{L} \subseteq\left(\bigcup_{r \in L} \widetilde{E}_{r}\right)_{[j]}, \quad \int_{\mathbb{T}} \widetilde{g}_{L}(x) d x=1, \quad \iint_{\mathbb{T}^{2}} \frac{\widetilde{g}_{L}(x) \widetilde{g}_{L}(y)}{|x-y|^{\alpha_{j}}} d x d y<K_{j} .
$$

Proof. Apply Lemma 3.1 repeatedly with $P$ every possible subset of $\Lambda$ with $n$ elements.

We need one further remark.

Lemma 3.3. Suppose $1>\delta>0$ and $E \in \mathcal{G}$. Then we can find $F \in \mathcal{G}$ with $d_{\mathcal{G}}(E, F)<\delta$ such that $F$ is the finite union of non-trivial closed intervals and there exist piecewise continuous positive $g_{j}: \mathbb{T} \rightarrow \mathbb{R}$ such that

$$
\int_{\mathbb{T}} g_{j}(x) d x=1, \quad \operatorname{supp} g_{j} \subseteq E_{[j]}, \quad \iint_{\mathbb{T}^{2}} \frac{g_{j}(x) g_{j}(y)}{|x-y|^{\alpha_{j}}} d x d y<K_{j}
$$

for all $j \geq 1$.

Proof. Let

$$
\Delta(x)=\max \left(0,2 \delta^{-1}\left(1-2 \delta^{-1}|x|\right)\right) .
$$

We know that there exist probability measures $\mu_{j}$ with

$$
\operatorname{supp} \mu_{j} \subseteq E_{[j]} \text { and } \quad \iint_{\mathbb{T}^{2}} \frac{d \mu_{j}(x) d \mu_{j}(y)}{|x-y|^{\alpha_{j}}} \leq K_{j},
$$


and we have chosen

$$
K_{j}>\iint_{\mathbb{T}^{2}} \frac{1}{|x-y|^{\alpha_{j}}} d x d y .
$$

Thus, if we set $F=E+[\delta / 2,-\delta / 2]$ and $g_{j}=\Delta * \mu_{j}$, we have the required result.

Proof of Lemma 2.7. By Lemma 3.3 it suffices to show that given $n \geq 1$, $\delta, \eta>0$ and $E$ satisfying the conclusion of Lemma 3.3, we can find an $F \in \mathcal{E}$ with $d(F, E)<\delta$.

Since $E$ contains non-trivial intervals, we can find an $m \geq 1$ such that $E_{[n+m]}=\mathbb{T}$. Write $E_{r}=E$ for $1 \leq r \leq n+m$,

$$
\Lambda=\{r: n+m \geq r \geq 1\}
$$

and, if $L \subseteq \Lambda$ contains $j$ elements with $n \geq j \geq 1$, set $g_{L}=g_{j}$.

Now choose $\widetilde{E}_{r}$ and $\widetilde{g}_{L}$ so that the conclusions of Lemma 3.2 hold. We set $F=\bigcup_{r=1}^{n+m} \widetilde{E}_{r}$. By Lemma 3.2(i),

$$
d_{\mathcal{F}}\left(E, \widetilde{E}_{r}\right)<\delta
$$

for all $r$ and so $d_{\mathcal{F}}(E, F)<\delta$. If we write $\Gamma$ for the collection of subsets of $\Lambda$ with exactly $n$ elements then

$$
\bigcup_{P \in \Gamma}\left(\bigcup_{r \in P} \widetilde{E}_{r}\right)_{[n]}=\left(\bigcup_{r=1}^{n+m} \widetilde{E}_{r}\right)_{[n]}=F
$$

so, by Lemma 3.2(iii),

$$
\bigcup_{P \in \Gamma} \bigcup_{I \in \mathcal{I}(P)} I \supseteq F \quad \text { and } \quad \sum_{P \in \Gamma} \sum_{I \in \mathcal{I}(P)}|I|^{\alpha_{n}+\eta}<\delta .
$$

Thus, if $F \in \mathcal{G}$, then $F \in \mathcal{E}$.

In order to show that $F \in \mathcal{G}$, we shall find piecewise continuous positive functions $f_{j}: \mathbb{T} \rightarrow \mathbb{R}$ such that

$$
\int_{\mathbb{T}} f_{j}(x) d x=1, \quad \operatorname{supp} f_{j} \subseteq F_{[j]}, \quad \iint_{\mathbb{T}^{2}} \frac{f_{j}(x) f_{j}(y)}{|x-y|^{\alpha_{j}}} d x d y<K_{j}
$$

for all $j \geq 1$. We split our task into three parts.

If $1 \leq j \leq n$, we set $f_{j}=\widetilde{g}_{\{1, \ldots, j\}}$ and use Lemma 3.2(iv), together with the observation that

$$
\left(\bigcup_{r=1}^{j} \widetilde{E}_{r}\right)_{[j]} \subseteq\left(\bigcup_{r=1}^{j} F\right)_{[j]}=F_{[j]} .
$$

If $n+1 \leq j \leq n+m$, we set $f_{j}=g_{j}$ and use Lemma 3.2(ii) to show that

$$
\operatorname{supp} f_{j}=\operatorname{supp} g_{j} \subseteq E_{[j]}=\sum_{r=1}^{j} E=\sum_{r=1}^{j} E_{r} \subseteq \sum_{r=1}^{j} \widetilde{E}_{r} \subseteq F_{[j]} .
$$


If $j \geq n+m+1$, we observe that the same calculation shows that

$$
\mathbb{T}=E_{[n+m]} \subseteq F_{[n+m]},
$$

so $F_{[n+m]}=\mathbb{T}$ and $F_{[j]}=\mathbb{T}$. We set $f_{j}=1$.

I should like to thank the referee for several improvements to the presentation.

\section{References}

[1] K. J. Falconer, The Geometry of Fractal Sets, Cambridge Univ. Press, Cambridge, 1986.

[2] K. Kuratowski, Topology, Vol. I, Państwowe Wydawnictwo Naukowe, Warszawa, and Academic Press, New York, 1966.

Centre for Mathematical Sciences

DPMMS

University of Cambridge

Clarkson Road

Cambridge CB3 0WB, UK

E-mail: twk@dpmms.cam.ac.uk

Received January 11, 2008

Revised version May 13, 2008 